\title{
The Research of Current Situation of Mechanical Innovative Design and Development Trend
}

\author{
Shao Shuai, Yan Pengcheng, Sun Jiangsheng, Zhang Lianwu \\ 18632823040@163.com \\ Mechanical Engineering College, Shijiazhuang 056003,China
}

Keywords: mechanical design; innovative design; TRIZ Theory

\begin{abstract}
In today's era of rapid development of science and technology, mechanical design industry has put forward higher requirements, and conventional mechanical design methods have been difficult to meet people's needs. Mechanical design is a scientific innovation to adapt to the times and to produce, it is possible to bring enormous social benefits and economic benefits, mechanical design always inseparable from innovation, only if we are good at design and innovation, can we produce more qualified and efficient product. This article briefly describes the content, process of the mechanical innovative design, summarize a variety of commonly used mechanical innovation design method; by plane wing evolution of TRIZ concepts were discussed; and finally briefly describes the meaning of Mechanical innovative design, and make a summary and outlook innovative mechanical design.
\end{abstract}

\section{Introduction}

"Innovation is the soul of a nation's progress, and is an inexhaustible motive force for national prosperity" $^{[1]}$. An industrial level is the cornerstone of the country's national economic development, in which the mechanical design is the most important, it can be said that any industry are not from open mechanical design. As it can be seen from the current situation of China's machinery industry in the design, general design dominate. While some foreign advanced industrial countries, have already begun to study the innovative design and come out from the original model of traditional design and encourage designers with new ideas, new principles and new capabilities to design new products. With a more open mind, an updated design mechanical design staff are more likely to succeed. A nation in terms of a country will be even more so. The level of innovation capability is an important indicator of the quality of talent. Related to national, corporate and personal future and destiny. Innovative mechanical design as an emerging discipline machinery industry, is a philosophy, cognitive science, innovation, aesthetics, scientific thinking in one of a modern science and technology $y^{[2]}$. If it can be developed and promoted, it will definitely have a significant impact to our machinery industry, it can certainly promote greater progress of China's industrial level.

\section{Mechanical Innovation Design}

\subsection{Connotation of Mechanical Innovation Design}

"Innovation" is the beginning of a word in economics, 1972, It was first proposed in Schumpeter's "Theory of Economic Development". Schumpeter pointed out that innovation is the invention of the first commercialization of the factors of production and production conditions to regroup production systems, as the invention is an important part of product innovation, design innovation is the core content ${ }^{[3]}$, therefore, it is self-evident that design innovation is important.

"Design" can be divided into three categories according to the degree of innovation: adaptive design, variant design and innovative design. Among them, the innovation design to provide important social value, innovative and unique design results in the field of design direction of the most challenging, is the highest goal pursued by the designer ${ }^{[4]}$.

The so-called mechanical innovative design (Mechanical Creative Design, MCD) refers to the designer's creativity into full play, has been associated with human scientific and technological 
achievements (including theories, methods, techniques, principles, etc.), innovative ideas, novel design creative and practical mechanism or mechanical products (device) a practice. It consists of two parts: First, improving and perfecting reliability, economy, applicability and so on of the technical performance or existing production machinery products of life, the second is to create a new machine design and new products to meet the new production or life need ${ }^{[5]}$.

No one discipline is completely independent in the knowledge-intensive highly contemporary society. Nowadays all disciplines have been very mature and complete. Mechanical innovative design is established based on existing mechanical design theory, many scholars machinery after a careful study of philosophy, cognitive science, innovation, aesthetics, scientific thinking, summarize its essence extracted for machinery industry, produced by the practice of the principle thus have mechanical innovative design this separate discipline. Birth and development of innovative mechanical design brings together the wisdom of mankind for thousands of years, it can be said products designed by mechanical innovative design are tried and tested, it must be science and technology and art combined with the product, not only innovative, practical, which also has a high aesthetic appreciation of the value ${ }^{[6]}$.

\subsection{The Study Object of Mechanical Innovation Design}

Mechanical design usually consists of three parts: First, the program structure design; second, motion design; Third, dynamic design. Among them, the first part of the creative need is the highest, while its design difficulty is also the largest ${ }^{[7]}$, conventional mechanical design usually only changes the size, power and other small design, it is difficult to have a substantial breakthrough. And with respect to the standard of conventional design, mechanical innovation design pay more attention to man in the design process, especially the leading and creative role in the design phase of the program structure. We concluded from the experience that mechanical inventions produced more in innovative mechanical structures.

\subsection{Process Analysis of Mechanical Creative Design}

The process of mechanical innovative design consists of four stages: determining the basic working principle of mechanic, carrying out the mechanism analysis and mechanical structure type synthesis, compositing the mechanism scale and movement parameter optimization, compositing mechanical motion parameter optimization, integrated mechanical dynamics and dynamic parameter optimization and, eventually tuning. Completing the following analysis and synthesis, it formed a mechanical creative design preferred embodiment ${ }^{[8]}$. Figure 1 is a general process of mechanical innovative design.

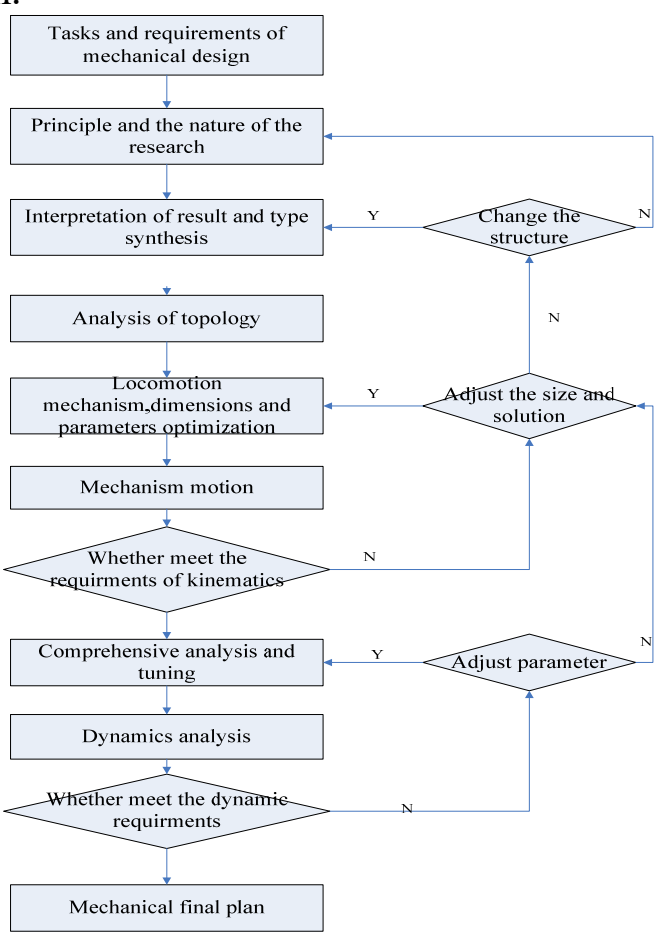

Fig.1 Flowchart Mechanical Innovative Design 


\section{Development Status and Common Design Methods in China and Abroad}

\subsection{Development Status at home and Abroad}

Nowadays the world has more than 20 innovative countries, including the United States, Britain, France, Germany, Japan and so on. Scientific and technological progress contribution rate refers to the amount of the share of GDP growth due to technological progress and growth is an important indicator of a country show innovative degree. Scientific and technological progress contribution rate of these innovative country is more than about 70\%, the United States and Germany is even up to $80 \%$. As early as 2005 , Comrade Hu Jintao has proposed an important stratetic thought to build an innovative country at the Fifth Plenary Session of the Sixteenth. March 2016 National "two sessions", China made it clear in 2020 , to the whole community research funding increased to $2.5 \%$, the contribution of scientific and technological progress reaches $60 \%$, to an innovative country and Talent into the ranks. Although there is a big gap with the world's leading countries, we are constantly catching up to them. Now it is the golden period of development, we must firmly seize the opportunity, we believe, it is not a dream to become an innovative powerhouse.

MCD is an created theories to be refined and summarized in various fields of industrial practice innovative approaches. Industrially developed countries of the Anglo-American is the most important mechanical innovative design and form a complete set of system theory, most of them from the existing mechanical structure will be summarized to form a theory and method. These countries started early, into a large, fast progress and have been successful in many industries. On one hand,on the practical application we invented many patented products rely on MCD, on the other hand, there are scholars began to attend to theoretical research. Today, in many journals you can see the literature on the MCD.

However, in terms of the world, although it has experienced a long-term research on mechanical innovative design and has made many achievements, there are still a lot of deficiencies of mechanical creative design, and there are still many problems to be solved:

1) the substance of the mechanical design innovation is the knowledge of innovative design essence, and now people have not formed a unified deeper understanding on innovative design.

2) the expression of the knowledge on mechanical creative design process. The discipline is a new one which derived the essence from various disciplines, there may be barriers to the communication between different disciplines, and is not conducive to the integration of knowledge.

3) Integration level of mechanical creative design process is not high. Mostly we just consider the design stage of the condition.

\subsection{Common Methods of Mechanical Creative Design}

Mechanical innovative design technology (MCD) is a branch of design method of disciplines and its development is not yet mature,together with mechanical design system (SD), computer-aided design (CAD), optimized design (OD), reliability design (RD), Tribology design (FD), finite element design (FED) and so on compose modern mechanical design methodology library ${ }^{[9]}$. There are many commonly used techniques and methods in mechanical innovative design. General introduction is below:

1) Discussion method

Discussion method (also known as brainstorming) is in a way of panel discussions whose size is generally from five to ten people, and stimulate divergent thinking, asking participants to emancipate the mind as much as possible, be free of thinking about the problem. Don't have to consider whether their idea is too ridiculous to be laughted at. Strive to create a relaxed atmosphere, which is more conducive to generate the innovative thinking. Their form of the organization refers to the following principles: (1) the discussants can speak freely, (2) don't criticize others ideas, (3) on other people's comments can not be premature,(4) everyone is equal, there is no principle of majority,(5) timely recording of other people ideas, discuss after sum up the most optimal results.

2) Analogy

Analogy is the comparative analysis of the similarity between two things, we seek innovation from the similarity to find solutions to problems. Analogy is extremely versatile. Such as bionics, the aircraft is invented based on the flight of birds,sonar is developed according to infrasound of 
dolphins and so on.

3) Enumeration method

Enumeration method is a method that enumerate the problems and help to adjust ideas, so as to carry on innovative design.

4) Transplantation

Transplantation is a method that the advanced technology of one product applied to another product, to design a new product. EDM technology is applied in mechanical processing which produced the EDM machine; vacuum technology is applied to the home appliances which invented the vacuum cleaner, shrink packaging machinery. This approach often produces breakthrough innovation of the functional principle ${ }^{[10]}$.

The following method is conducive to open mind, enhance creativity, but it can also have drawbacks, in fact, discussion method is trial and error, analogy and transplantation is not applicable to all products, and enumeration method includes heavy workload, sometimes we can not reach intended purpose. Thus it gave rise to the principles and methods of TRIZ integrated inventions ${ }^{[11]}$.

\section{TRIZ Theory Introduction and Application Examples}

\subsection{TRIZ Theory Introduction}

Innovative design theory TRIZ (theory of inventive problem solving) is the prefix of the Russian invention theory, the theory is put forward by the former Soviet Union -inventor Genrich Altshuller (GSAltshuler) and others from 1946,based on the study of 2.5 million patent ${ }^{[12]}$. The former Soviet Union with the support of this theory, the military industry produced a qualitative leap. Therefore, it has been the secret of former Soviet Union state until the mid-1980s to spread to the West, and has been promoted in some enterprises which access to a large number of patents and it has brought great wealth.The inventor of TRIZ from the outset consider that the invention issue follow a common principle, this principle can be abstracted from every design domain, and then applied to various design fields to guide the design of different areas. TRIZ is a systematic theory to solve the invention problem based on knowledge (knowledge-based), human- oriented(human-oriented). It makes the core of product innovation, the process of creating new works specific, it makes a number of rules, algorithms and principles of the invention for designers to use, and has become a more complete design theory ${ }^{[13]}$.Currently, it has been widely used in the United States, Europe, Japan, Korea and other countries and regions. It was not introduced into China until 1988, and increasingly catch the attention of government departments and researchers.

Main contents of TRIZ include:

1) Evolutionary theory of product

The evolution of the product will be divided into four stages of infancy, growth, maturity and out of phase, research products evolutionary patterns, evolution and the evolution of the law of course.

2) Analysis of problems

It includes functional analysis of products ,the determination to the ideal solution, analysis of available resources and determination to the conflict areas, the analysis is an important step to solve the problem.

3) The principles of conflict resolution

The main research are technical and physical conflict, to solve technical conflict with the 40 principles of the invention, to solve the physical conflict with separation principle.

4) Object - field analysis

All functions can be decomposed into two substances and one field, based on matter - Transform field model, it offers 76 standard solutions.

5) Effect

It refers to the application of the relevant laws of this field especially other areas to solve design problems, such as the use of mathematics, chemistry, biology and other electronics in principle to solve the mechanical design innovation.

6) Algorithm for inventive problem solving 
Algorithm for Inventive Problem Solving ARIZ (algorithm for inventive problem solving) using a logical process gradually stylized initial questions, such that the initial question the most fundamental conflict is clearly exposed, to facilitate problem solving ${ }^{[9]}$.

TRIZ theory is actually a comprehensive theory that combines various methods of invent, analyze ideas and algorithms to solve the technical problems and achieve an innovative design. Figure 2 illustrates the architecture of this theory ${ }^{[14]}$.

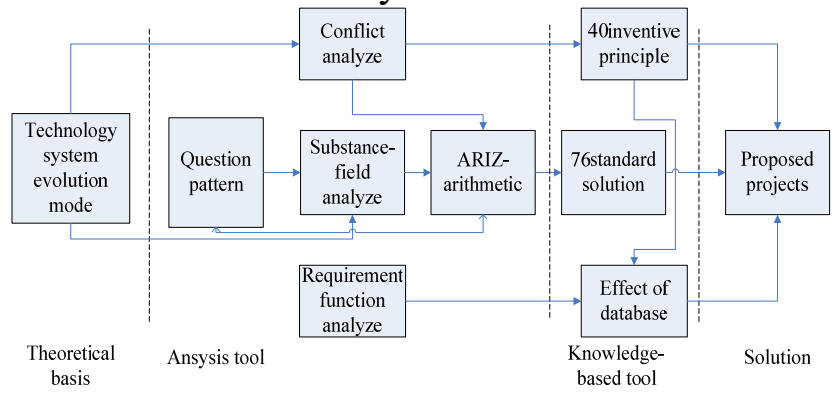

Fig.2 TRIZ architecture diagram

\subsection{TRIZ Theory Examples}

TRIZ theory analysis to solve the problem are systematic and comprehensive, Figure 3 shows TRIZ problem-solving in detail ${ }^{[15]}$.

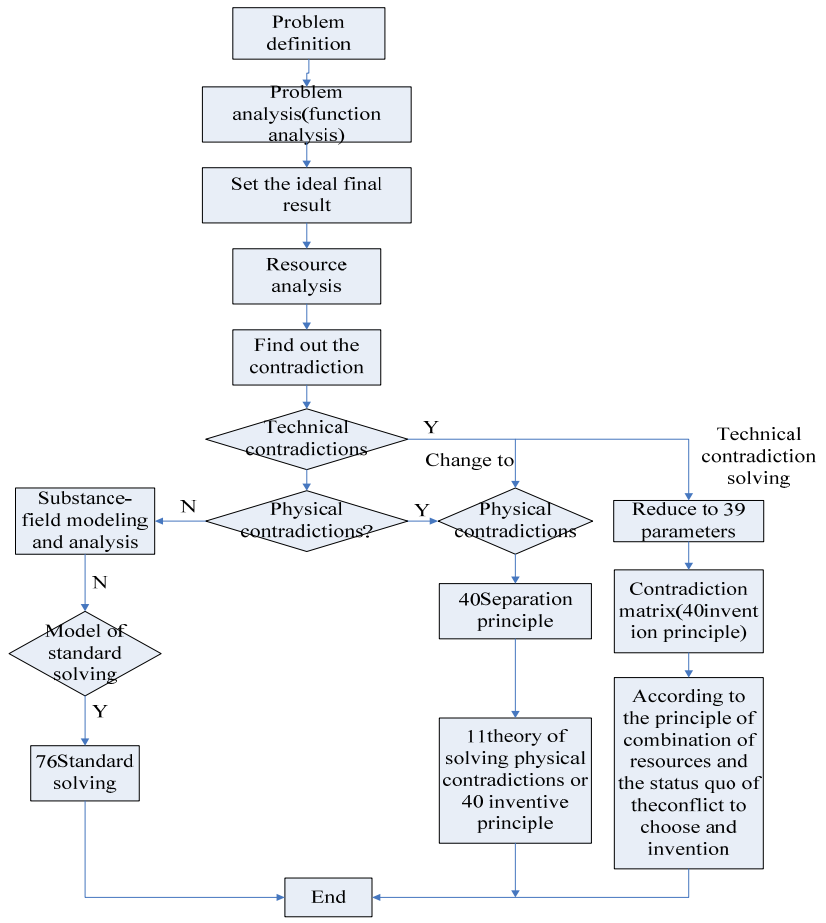

Fig.3 TRIZ problem-solving steps

It is the evolution of aircraft wings as an example to introduce the methods of TRIZ analysis: Early aircraft wings are flat. Initially they are rectangular wings and are easy to manufacture. But because of its wide wings, they will bring the aircraft resistance, seriously affecting the speed of the aircraft. After trapezoidal wings were developed, it greatly enhanced the speed of flight. Then, starting from the Western countries, the jet plane went to heaven one after another, stepping into the jet age, the speed of flight is approaching the sound velocity soon. However, the "shock" occurs on the wings on high-speed condition to make the surface pressure of wings changes, wind resistance at this time is ten or even more times than lower speed, this phenomenon is called "sound barrier." In order to overcome the problem of "sound barrier", many countries are trying new wings. German scientists first discovered that the wings designed like swallows swept-back wings may delay "shock" generation and reduce the "sound barrier" phenomenon. But the lift force of swept-back wings is smaller than the not swept-back wings in the same circumstances. It is unfavorable for the 
aircraft to take off, land and cruise, it's not only a waste of fuel but increasing the instability of flight safety. Therefore, it is a big problem for the aviation industry to design a wing that adapts to all speed.

Using technology contradictions to analyze the problem: speed improving and movement of objects increasing in energy consumption is a contradiction. After considering the selection principle 15 (dynamic), principle 35 (parameters) two principles of the invention ${ }^{[16]}$.

Changing the form of the aircraft flight, the plane shape can be different in different flight state, it can save a lot of unnecessary energy consumption. According to the 35 principles,parameters changes of objects and the 15 principles,objects dynamic, we gain enlightenment to make the wings of an airplane to be moving parts. Using flat wings when takeing off and landing, delta wings during sustained flight, so that we not only enhance the lift at low speed, but reduce resistance of wings at high speed, thereby reducing energy consumption in two conditions ${ }^{[17]}$. The problem is resolved, and the first frame variable-geometry wing fighter aircraft F111 was born.

\section{Summary of the Innovative Design of Mechanical and Prospects}

Theoretical study of mechanical innovative design will be able to bring huge economic and social benefits. We summarize and prospect from the following points :

1) In-depth study of mechanical innovation design, it will provide strong theoretical support to create new mechanical design.

2) The research of mechanical innovation design is more refers to the changing of mind during human's invention, while the progress of human thought, the mechanical "expert" system will be more intelligent, thus promoting the mechanical design industry towards the more intelligent, automation, optimization, integrated direction.

3) Mechanical products of innovative design, will repeated scrutiny in the stage of principle determination stage before deciding down, structural design and dimensions design will be more creative, so the products must contain a higher value-added of key technology, so that the product not only has superior performance, but also has superior styling performance. It is the perfect combination of art and the principles of science and technology, it's good coordination of human-machine systems, and also a good economy and so on. So there is no doubt that it improves the competitiveness in similar products, especially when the patented product technologies form industrialization, it can create a higher economic and social benefits.

4) It cultivates the creative thoughts of designers in the practice of mechanical innovative design, enhances the ability to innovate and improves people's self-awareness of innovative design and technical feasibility to make the mechanical innovative design as a tool or means,thus promoting prosperity and updates of new products, shortening the update time of new products, to create more wealth for the community.

\section{Conclusion}

We briefly introduce the status and development trend of mechanical innovative design in this article, we should recognize clearly that our country is still a developing country, we have a long way to go in industry, particularly mechanical design to catch up with the world's advanced countries. mechanical Innovative design is the only way to catch up with the world's advanced industrial country, and it is also full of difficulties. we hope this paper can play a little reference to you, and more scholars and experts pay attention to mechanical innovative design, and make a breakthrough in this industry.

\section{References}

[1] Huang Wan, Wang Donglin. Innovative of Mechanical Design[J]. Mechanical Design and Manufacturing, 2004(5): 51-52.

[2] Song Zhiqiang Research on Examples and Practice of Mechanical Innovative Design[J]. 
Hulunbeier College,2008,16(1): 96-99.

[3] You Xiaohong, Lei zhuFeng Research and Development of Mechanical Innovative Desig $\mathrm{n}[\mathrm{J}]$. Mechanical Management and Development, 2011(6): 58-59.

[4] Teng Qi, Song Kezong, Wang Hongyan. Research on Mechanica Innovative Design[J]. Forging Machinery, 2002(3): 6-9.

[5] Shen Huiping Research and Development of Mechanical Innovation Design[J].Mechanical Science and Technology, 1997,16(5): 791-795.

[6] Jin Zhongyu, Chen Gang Discussion of TRIZ Theory Applied in Mechanical Creative Practice[J] Heilongjiang Science, 2012,3(2): 49-52.

[7] Wei Yuxin. Innovative Methods Research of Modern Mechanical Design[J]. Equipment Manufacturing, 2009(5): 173-174.

[8] Tan Runhua. TRIZ and Application: Technical Innovation Process and Methods[M] Higher Education Press, 2010,11.

[9] G. S. Altshuller.TRIZ, SYSTEMATIC INNOVATION AND TECHNICAL CREATIVITY. Huazhong University of Science and Technology Press, 2008,10.

[10] Han Qing. Innovative Design theory and methods of Modern Mechanical Design[J]. Jiangxi Building Materials, 2015(8): 50-51.

[11] Chen Tong. Research and Innovation of Modern Machine Design Technique[J]. Machanical Research \& Application, 2015(2): 195-197.

[12] Yuan Feng, Wang Taiyong, Nie huijuan. Innovative Design of Mechanical Products Feature and Principles based on innovative design theory/functional analysis[J]. Computer Integrated Manufacturing System, 2006,12(2): 204-209.

[13] Tan Runhua. Development Issues Product Innovation[J]. Journal of Mechanical Engineering, 2003,39(9): 11-14.

[14] Saeed Moaveni [US]. Finite Element Analysis Theory and Application -ANSYS second edition [M]. Electronic Industry Press, 2005.

[15] Deng Yuanchao, Liu Xiaopeng. Teaching Discussion of TRIZ in "Mechanical Creative Design" Course[J]. Education Forum, 2014 (45): 157-159.

[16] Yang Hairong. Innovative Methods of Modern Mechanical Design[J]. Design and Analysis, 2011 (18): 161-162.

[17] Zhu Youwei. Mechanical Innovative Design[J]. Dandling Equipment, 2006(12): 16-18. 